\title{
Análisis técnico-económico de la erosión del suelo y su impacto en el bienestar de productores
}

\section{Technical-economic analysis of the soil erosion and its impact on the well-being of producers}

OMAÑA-SILVESTRE, José Miguel $*^{+}{ }^{1}$, QUINTERO-RAMIREZ, Juan Manuel ${ }^{2}$ y OMAÑASILVESTRE, Juan Carlos ${ }^{3}$

${ }^{1}$ Posgrado en Economía, Colegio de Posgraduados, Campus Montecillo, Texcoco, Estado de México, México

${ }^{2}$ Cátedra CONACyT Ciudad de México, México

${ }^{3}$ Facultad de Ciencias Agropecuarias, Universidad Autónoma del Estado de Morelos, Cuernavaca, Morelos, México

ID $1^{\mathrm{er}}$ Autor: José Miguel, Omaña-Silvestre / ORC ID: 0000-0002-5356-549X, CVU CONACYT ID: 59890

ID 1 ${ }^{\text {er }}$ Coautor: Juan Manuel, Quintero-Ramírez / ORC ID: 0000-0002-1040-2690, CVU CONACYT ID: 292056

ID $2^{\text {do }}$ Coautor: Juan Carlos, Omaña-Silvestre / ORC ID: 0000-0001-6096-1864, CVU CONACYT ID: 374243

DOI: $10.35429 / J U S D .2019 .15 .5 .1 .12$

Recibido: 30 de Marzo, 2019; Aceptado 30 de Junio, 2019

\section{Resumen}

La erosión de suelos en comunidades rurales agrícolas de México y los bajos rendimientos, es el tema en el presente trabajo, localidades en donde se ha dejado de producir debido a la baja rentabilidad y productividad generadas por la pérdida de nutrientes a consecuencia de fenómenos naturales, así como por la acción del hombre. El Objetivo es identificar la condición actual del suelo y su impacto en la producción agrícola, con el fin de sugerir técnicas de mejoramiento y conservación del suelo apropiadas a las características regionales, para elevar el nivel de ingresos de los productores de una comunidad rural de Hidalgo, México. El trabajo se divide en tres etapas: la primera consistió en identificar los cultivos predominantes agrícolas; la segunda, toma de muestras para elaborar el análisis de suelo; la tercera, evaluar económicamente los ingresos que actualmente generan las actividades agrícolas que se practican en la comunidad y posteriormente evaluar los cambios que se podrían generar al aplicar medidas de conservación de suelos; así como la aplicación de la metodología para identificar la rentabilidad y de los productos.

Suelo Agrícola, Erosión, Conservación de suelos

\begin{abstract}
Soil erosion in rural agricultural communities in Mexico and low yields is the subject of the present work, places where production has stopped due to the low profitability and productivity generated by the loss of nutrients as a result of natural phenomena, as well as by the action of man. The objective is to identify the current condition of the soil and its impact on agricultural production, in order to suggest soil improvement and conservation techniques appropriate to regional characteristics, to raise the income level of producers in a rural community of Hidalgo, Mexico. The work is divided into three stages: the first consisted of identifying the predominant agricultural crops; the second, sampling to develop the soil analysis; the third, to evaluate economically the income that currently generates the agricultural activities that are practiced in the community and later to evaluate the changes that could be generated when applying measures of soil conservation; as well as the application of the methodology to identify the profitability and the products.
\end{abstract}

Agricultural Soil, Erosion, Soil conservation

Citación: OMAÑA-SILVESTRE, José Miguel, QUINTERO-RAMIREZ, Juan Manuel y OMAÑA-SILVESTRE, Juan Carlos. Análisis técnico-económico de la erosión del suelo y su impacto en el bienestar de productores. Revista del Desarrollo Urbano y Sustentable. 2019. 5-15: 1-12

\footnotetext{
*Correspondencia al autor (correo electrónico: miguelom@colpos.mx))

$\dagger$ Investigador contribuyendo como primer autor
} 


\section{Introducción}

Por su ubicación geográfica, su topografía y sus climas, los suelos del territorio mexicano son complejos, pues se encuentran al menos 26 de los 30 grupos reconocidos por el Sistema Internacional Base Referencial Mundial del Recurso Suelo (FAO, ISRIC y ISSS, 1998). Por su extensión destacan: los Leptosoles (28.3\% del territorio), Regosoles (13.7\%), Phaeozems (11.7\%), Calcisoles (10.4\%), Luvisoles (9\%) y Vertisoles $(8.6 \%)$, que en suma cubren $81.7 \%$ del país (SEMARNAT, 2008). El Territorio nacional comprende 198 millones de hectáreas conformadas en $15 \%$ de tierras agrícolas, $58 \%$ de tierras de agostadero y esparcimiento, $23 \%$ de bosques y selvas, y el restante $4 \%$ en cuerpos de agua y asentamientos humanos. (INEGI, 2003)

La evaluación desarrollada por la SEMARNAT en 2001-2002, señala que al menos $45 \%$ del suelo del territorio nacional ha sido afectado por algún tipo de degradación, del cual $5 \%$ presenta una condición de deterioro de severo o extremo y el $95 \%$ se ubica dentro de los márgenes de ligero o moderado. Las principales causas de erosión son debido al cambio de uso de suelos hacia la agricultura y el sobrepastoreo que tienen que ver directamente con la reducción de la cobertura vegetal que tiene como función la conservación del suelo. (SEMARNAT, 2003)

La erosión significa la pérdida progresiva que se produce en los terrenos debido a la acción física, química del agua, viento y agentes biológicos. Es el acarreo de partículas y de elementos nutritivos que lo forman (arenas, limo, arcilla nitrógeno, potasio, etc.) en grado mayor o menor ocurre, desde las partes más altas, hacia los valles donde se acumulan como azolves, esos componentes de suelo benefician si el material acarreado es rico en elementos nutritivos y los perjudica cuando lo constituyen arenas, escorias, tepetates o cualquier otro componente estéril. (Becerra, 1999).
La erosión como fenómeno de transporte de elementos contenidos en los suelos genera condiciones de deficiencia de nutriente en parcelas agrícola representa un gran problema para comunidades dedicadas a la agricultura de temporal, la SEMARNAT (2003), en el documento de ordenamiento ecológico indica que el estado de Hidalgo presenta tres zonas con potencial de erosión la primera al norte en los municipios de Cardonal y Santiago Anaya (uso de suelos, agrícola de temporal) zona que está dominada por pastizales y agricultura de temporal ambos ubicados en el eje neovolcánico y la sierra madre.

La segunda al sur del estado, los municipios de San Agustín Tlaxiaco y El Arenal (uso del suelo es agrícola de temporal y agostadero). La tercera y última zona se localiza en los Llanos de Apan, incluyendo al municipio del mismo nombre (uso potencial del suelo en Apan es en su mayoría agrícola; presenta un 64\% de temporal) y Almoloya (uso potencial del suelo es en su mayoría agrícola, teniendo una producción de maíz, cebada y frijol en un $76.12 \%$ de la superficie municipal), limítrofe con el estado de Puebla, municipios que presentan un alto potencial de erosión debido a las actividades de agricultura y pastoreo. El municipio más afectado en Hidalgo es el Cardonal, ubicado en el norte del territorio.

La presente investigación se desarrolla en la comunidad de Cuesta Blanca, Municipio de Cardonal en Hidalgo, México; lugar donde se realizan una diversificación de cultivos y que en los últimos años han disminuido constantemente los rendimientos y a los productores les ha afectado directamente en sus ingresos, lo que han buscado nuevas técnicas para poder incrementar sus cosechas sin perjudicar las propiedades del suelo.

Con el estudio de suelos en la zona de estudio y con la aplicación de la metodología de Matriz de Análisis Política, se podrá identificar que propiedades son necesarias para poder hacer más fértil los suelos y que los cultivos aumenten los rendimientos, así como conocer la rentabilidad generada a partir de la aplicación de nuevas técnicas en el tiempo. 
Se pretende dar una propuesta para poder mitigación de la erosión de esta comunidad con la aplicación de ciertas actividades agrícolas que puedan reducir este proceso y que además pueda dar nutrientes perdidos al suelo. Por otra parte, con la aplicación de nutrientes al suelo, identificar la rentabilidad de los cultivos para generar un análisis de costos para determinar el imparto generado de las practicas realizadas al suelo a ciertos cultivos que se practican a la comunidad.

La implementación de técnicas de conservación y mejoramiento de suelos, pueden mejorar las condiciones de suelo que tienen una baja rentabilidad de cultivos básicos, lo que afecta a la economía del productor.

\section{Metodología}

El trabajo de campo estuvo dividido en tres etapas: la primera consistió en la identificación de la zona de estudio en cuanto a características como tipos de suelos, vegetación, relieve (\% de pendientes), cultivos utilizados, tipo de agricultura y condiciones susceptibles para la aplicación de alternativas de conservación de suelos; la segunda fue la toma de muestras para elaborar el análisis de suelo; la tercera etapa consistió en el levantamiento de cuestionarios a productores para evaluar económicamente los ingresos que actualmente generan las actividades agrícolas que se practican en la comunidad aplicando la metodología de la Matriz de Análisis de Política y, posteriormente evaluar los cambios que se podrían generar al aplicar dos medidas de conservación de suelos.

\section{Muestreo y análisis de suelo}

Para el análisis del suelo se determinaron cuatro puntos en los que se tomaron muestras, los cuales fueron seleccionados mediante la evaluación de sus condiciones fisiográficas. El primer punto, ubicado en la latitud $20^{\circ} 38^{\prime} 51^{\prime \prime}$ y longitud 99 $04^{\prime} 10^{\prime \prime}$, con una altitud de 2,086 msnm, son terrenos agrícolas con una pendiente de $10 \%$ hasta $50 \%$, la vegetación predominante es el agave, los cultivos predominantes son el maíz y frijol. Presenta floración poco abúndate de roca caliza, la profundidad de los suelos va de 30 a $60 \mathrm{~cm}$.
El segundo punto, con una latitud $20^{\circ} 38^{\prime} 14^{\prime \prime}$ y longitud $99^{\circ} 04^{\prime} 30^{\prime \prime}$ con una altitud de 2,082 msnm son terrenos agrícolas con pendientes de $3 \%$ y $8 \%$, la vegetación predominante de agaves como límite entre parcelas, matorrales y pequeñas parcelas de nopal tunero, los cultivos predominantes en los últimos ciclos han sido el maíz y el frijol de variedades criollas. El tercer punto, con una latitud de $20^{\circ} 38^{\prime} 53^{\prime \prime}$ ' y de longitud $99^{\circ} 05^{\prime} 15^{\prime \prime}$, con $2,128 \mathrm{msnm}$, pendientes de entre $10 \%$ y $20 \%$, son terrenos agrícolas con vegetación predominante de pinos, nogales, agaves y matorrales, estos últimos con funciones de límites y cercado entre las parcelas, los cultivos implementados en los últimos ciclos agrícolas cebada maíz y frijol.

Y finalmente el cuarto punto, latitud $20^{\circ} 39^{\prime} 14^{\prime \prime}$ y longitud 99 $04^{\prime} 20^{\prime \prime}$, a 2101 msnm, con pendientes que van de $5 \%$ hasta un 90\% esto ya en cercanías con las barrancas, suelos de tipo leptosoles líticos con afloración abundante de roca caliza, límites con predominancia de pino piñoreno y enebros, abundancia de agaves como cercas vivas entre parcelas, los cultivos de maíz y frijol.

Los materiales utilizados fueron palas, cubetas y una manta para hacer la combinación de las muestras recolectadas en el punto muestreado. Para la toma de muestras de suelos, se cavaron hoyos en los terrenos (melgas) aproximadamente de $50 \mathrm{~m}^{2}$, del cual se extrajeron de 10 a 15 sub-muestras siguiendo un camino en zigzag a fin de abarcar todo el lote. Cada submuestra fue tomada a una profundidad de $30 \mathrm{~cm}$.

$\mathrm{Al}$ terminar la toma de las sub-muestras se mezcló todo el contenido del suelo y haciendo una reducción de volumen de muestras hasta tener aproximadamente $1 \mathrm{~kg}$ de mezcla. Al final se dejó secar totalmente al aire libre. Las cuatro muestras compuestas fueron enviadas al laboratorio como muestras representativas de los lotes. El muestreo del suelo se llevó a cabo con el apoyo del laboratorio de análisis de suelo, agua y composta de, la Secretaría de Desarrollo Rural y Equidad para las Comunidades (SEDEREC). 


\section{Evaluación económica de los sistemas de producción}

Para la evaluación económica de los sistemas agrícolas se aplicó de Matriz de Análisis de Política (MAP), con el fin de medir la rentabilidad y eficiencia de la producción de los cultivos de maíz, frijol y cebada en la comunidad de Cuesta Blanca. Se aplicaron cuestionarios a productores, en las que se abordan temas como tipos de cultivos empleados, costos de producción, datos históricos de producción. Se tomó como parámetro los datos en base a una hectárea, para determinar indicadores de rentabilidad, producción y costos.

La Matriz de Análisis de Política (MAP) desarrollada por Erick Monke y Scott R. Pearson (1989), de las Universidades de Arizona y Stanford, es una herramienta analítica para identificar la problemática de los sistemas de producción y tomar decisiones de política. El análisis se complementa con la metodología de análisis de Ingreso y de Inversión del Instituto de Desarrollo Económico del Banco Mundial.

El trabajo principal de la MAP, es construir las matrices de ingresos, costos y ganancias (a precios privados y económicos) en los sistemas de producción regionales, con base a la información obtenida directamente en las diferentes etapas de la cadena productiva. El método permite obtener los niveles actuales de ingresos, costos y ganancias que los productores de una región están obteniendo en los sistemas de producción (patrón de cultivos), y aquellos que obtendrían si ellos recibieran ingresos por ventas y pagos de costos de producción basados en precios que asignan los recursos de manera eficiente (o eliminar subsidios e impuestos).

Los precios privados son los precios de mercado actuales, mientras que los precios de eficiencia económica, también llamados sociales, son aquellos que reflejan valores de escasez o costos de oportunidad económica. Estos últimos corrigen los efectos de políticas distorsionantes, esto es, de políticas que originan un uso ineficiente de los recursos.

Una identidad determinada en la MAP, mide la ganancia como la diferencia entre ingresos y costos de producción:

$$
\text { Ganancias }=\sum P_{i} X_{i}-\left|\sum P_{j} Y_{j}+\sum P_{k} Z_{k}\right|
$$

donde:

$P i$ : precio del producto en el mercado nacional

$X i$ : cantidad de toneladas producidas por hectárea

$P j$ : precio de los insumos comerciables en el mercado nacional

$Y j$ : cantidad de insumos comerciables e indirectamente comerciables aplicados por hectárea

$P k$ : precio de los factores internos en el mercado nacional

$Z k$ : cantidad de factores internos aplicados por hectárea

\section{Análisis del ingreso}

El valor de la producción (ingreso total) para una explicación más profunda, se puede desagregar en sus dos componentes principales: consumo intermedio y valor agregado.

Consumo Intermedio: Es el gasto del sector agrícola en otros sectores de la economía regional. Se compone de los gastos corrientes de la finca (exceptuando salarios) y el pago por la utilización de los equipos de maquinaria agrícola y bombeo. Se obtiene deduciendo del valor de la producción o ingreso total (rendimiento por precio del producto) todos los pagos por productos provenientes de otras fincas y de empresas industriales.

Valor agregado: Es la contribución de la actividad agrícola a ingreso del propio sector. Es el monto del ingreso total de la finca utilizado para pagar a los recursos mano de obra, tierra y agua, capital y la administración. Se obtiene al descontar de los ingresos totales o valor de la producción (rendimiento por precio del producto) el consumo intermedio, es decir, los gastos corrientes y el pago por la utilización de la maquinaria agrícola y equipos de bombeo. 


\section{Medidas para mitigar erosion}

Utilización de presas de rama, con base en las condiciones del lugar que a través del recorrido de campo, se determinaron pendientes y zonas en las que requiere ser atendidas con este método para controlar los escurrimientos y arrastre de suelo por las fuertes lluvias determinando número de presas mediante las condiciones de pendiente y de cárcavas encontradas en campo, la disponibilidad de materiales en campo para la posible aplicación de la alternativa es favorable ya que en el sitio existes matorrales y desechos de ramas.

Abonos orgánicos fermentados tipo Bocashi, en la visita de campo se indago sobre la disposición y costos de dichos ingredientes para la generación de una tonelada de este insumo, para una hectárea, encontrándose que existen condiciones favorables en la comunidad, en existencia y bajo costo.

La aplicación de un biofertilizante es una alternativa de solución al problema de disponibilidad de nutrientes para los cultivos, la cual permitirá mejorar gradualmente la fertilidad, la nutrición y un mayor rendimiento en número de plantas por hectárea, ya que estos biofertilizantes son fuente constante de materia orgánica ayudan a nivelar las condiciones de $\mathrm{pH}$, con la intención de llevarlo a un estado acido en el cual pueda existir un flujo de elementos, para que estos puedan ser absorbidos por los cultivos empleados, y así que pueda generar una mayor rentabilidad económica por área cultivada.

\section{Resultados}

\section{Niveles de pendiente}

A través de los perfiles de elevación, una herramienta de Google Earth@, se determinaron los niveles de pendiente. Las líneas son rutas marcadas sobre la delimitación de la comunidad que se establecieron con dirección del escurrimiento más importante con la finalidad de conocer el relieve de la comunidad y la dirección de los escurrimientos que pudiesen formar (Figura 1).
La línea 1 que se marca sobre la zona de estudio indica que la elevación va desde $2 \%$ hasta $77 \%$ como máxima. Con una altura máxima de 2,143 msnm y una mínima de 2,057 msnm en la que intervienen zonas de cultivo descubiertos de vegetación la mayor parte del año, entre parcela y parcela se encuentran cercos con agaves para evitar el movimiento de suelos conforme a la pendiente, que en su parte final la pendiente es muy pronunciada debido a que llega una ladera. La línea 2 marca una pendiente de $5 \%$ y máxima $69.8 \%$ de inclinación en la parte final de la línea que termina en una ladera, cruza por una zona de cultivos que esta descubierta de vegetación la mayor parte del año con cercos vivos de agave y montículos de tierra, con una altura de 2,114 msnm y la más baja de 2,059 msnm conforme se desciende de las faldas del cerro.

De igual forma, la línea 3, indica pendientes que van del $20 \%$ hasta un $70.6 \%$ como máxima de inclinación en la parte final de la línea debido a que termina en una zona de laderas, con una altura de 2,085 msnm y 2,059 msnm como mínima, atraviesa zonas de cultivos descubiertas de vegetación la mayor parte del año con cercos vivos de agave $y$ cactáceas. La línea 4, muestra una pendiente máxima de hasta $10 \%$ y mínima de $7 \%$ siendo la parte más plana donde en los terrenos descubiertos la mayor parte del año no hay vegetación debido a que se han adecuado para la actividad agrícola, con alturas de 2,096 msnm máxima y 2,078 msnm como mínima, tiene como cercos vivos agaves y matorrales para evitar el movimiento de suelos.

De igual forma la línea 5, tiene pendientes de $3.5 \%$ como mínima y una máxima de $4.8 \%$ las variaciones de pendiente son mínimas debido a la adecuación de los terrenos para cultivos comúnmente desprovistos de vegetación la mayor parte del año en donde predominan como cercos vivos los agaves y matorrales, con alturas de 2,081 msnm y 2,090 msnm. Por el contrario, la línea 6 muestra pendientes de $24 \%$ hasta un $29 \%$ como máxima donde hay aproximación a la ladera más cercana, la línea cruza terrenos agrícolas descubiertos de vegetación, con cercos vivos como agaves y cactáceas, con elevaciones de $2052 \mathrm{msnm}$ hasta $2083 \mathrm{msnm}$. 
Y finalmente la línea 7 trazada sobre el poblado indica pendientes de $5 \%$ hasta un $8 \%$ como máxima donde los terrenos de cultivos son planos esto debido a la adecuación del terreno para la actividad agrícola con la gran parte del año sin vegetación, donde de igual forma prevalecen los cercos vivos de agave y una altura de $2063 \mathrm{msnm}$ hasta $2080 \mathrm{msnm}$.

La Figura 1, muestra el comportamiento de las líneas trazadas sobre la superficie de la comunidad las pendientes son muy pronunciadas en los límites de la comunidad, en donde comienzan los bosques de pino en la que las pendientes llegan hasta un $77 \%$ de inclinación y en zonas con un mínimo de $2 \%$ en las que existen terrenos agrícolas adaptados para la agricultura donde se tiene cercos de agave con la finalidad de detener el suelo arrastrado por agentes como el agua que lo llevan cuesta abajo, presenta una altura máxima de 2,143 msnm en la primer línea trazada sobre el polígono del poblado.

Con lo anterior se determina cuáles son los lugares que pudieran sufrir procesos de erosión hídrica esto debido al nivel de pendiente y condiciones de vegetación predominante, lo que demuestra que el suelo se encuentra en todos los puntos sin vegetación la mayor parte del año y solo en los límites se encuentran montículos de tierra y cercos vivos de agave y cactáceas, puntos en los que se pudieran implementar las presas de rama acomodada para detener el flujo del suelo con el fin de reducir el transporte de sedimento pendiente abajo.

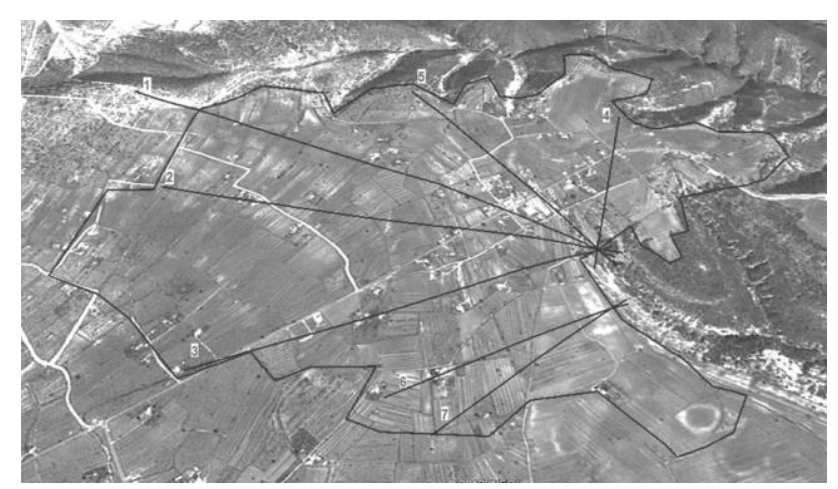

Figura 1 Polígono de la comunidad Fuente: Elaboración propia Google Earth@

\section{Descripción de los suelos en la comunidad y su condición actual}

Dentro de la zona de estudio, los suelos son de tipo leptosoles, leptosoles líticos y los molisoles siendo los más abundantes, con afloraciones de roca caliza en la mayor parte de la comunidad, la vegetación que predomina en las partes más planas es el agave que es utilizado como barra de contención de suelos así como cerca viva y límite entre las parcelas de cultivo, y de forma silvestre se reconoce la abundancia de matorral, pinos (piñoneros) en zonas con pendiente de hasta $90 \%$, enebros en las partes con menor pendiente son aquellas que representa entre el $3 \%$ y el $8 \%$. Dentro de los límites de la comunidad se encuentra con bosques de pino y enebro mismos que en determinados puntos se asocian. La existencia de otro tipo de especies de árboles es debido a la acción humana. Existen pequeñas parcelas con dominancias de nopal tunero, así como la de xoconotztle.

\section{Resultado de muestreo de suelos}

Los resultados de los análisis de suelos enviados al Laboratorio Móvil de Análisis de Suelo, Agua y Composta del Distrito Federal (Tabla 1), muestran que tienen una textura dominante para los cuatro lugares muestreados de tipo arcilloso (con valores que van de $33.80 \%$ al $53.24 \%$ ) seguido de arena (con un porcentaje que va del $28.76 \%$ al $58.76 \%$ ) y de limo con un reducido porcentaje (que va del $7.44 \%$ al $27.64 \%$ ), lo cual da a los suelos de la comunidad una porosidad favorable para la aireación y flujo de agua debido al volumen de las dos primeras partículas, en el primer muestreo se presenta una textura de tipo franco arcillo arenoso, en el segundo sitio tenemos franco arcilloso, en el tercer sitio es de tipo arcilla y por último el cuarto lugar tiene arcilla arenosa.

El $\mathrm{pH}$ que se tiene en promedio es de 7.56 que lo hace medianamente alcalino esto debido a los niveles dependientes en los que se localizan los terrenos agrícolas el dato más alto pertenece a la muestra número 3 con $7.81 \mathrm{y} \mathrm{el}$ más bajo de 7.15 en el sitio dos que se mantienen neutros. Cuando se tienen valores de $\mathrm{pH}$ ligeramente alcalinos como es el caso, lo mejor es acidificarlos hasta valores cercanos a un intervalo 5.6-6.2 dado que los elementos se tornan lábiles; al respecto el efecto de Bocashi (Kononova, 1982). 
El abono orgánico fomentado tipo Bocashi brindara a los suelos un $\mathrm{pH}$ de entre 6.0-7.5 generando una actividad microbiana positiva (Restrepo J., 2006). La comunidad presenta suelos con un $\mathrm{pH}$ alcalino lo que pudiera generar condiciones en las que los elementos contenidos en el suelo, no puedan ser tomados por los cultivos, que durante los últimos ciclos agrícolas han presentado un déficit en la producción de los cultivos empleados.

La conductividad eléctrica (CE) presentó valores de 0.48 a $0.52 \mathrm{dS} \mathrm{m}^{-1}$, por lo que no se considera salino (Rechards, 1985), con efectos despreciables de salinidad influenciado por los niveles de $\mathrm{pH}$. De materia orgánica (MO) se tienen desde 6.3 que representan niveles medios y en la última de las muestras presenta $13.1 \%$ de nivel alto.

La concentración de los elementos Nitrógeno $\left(\mathrm{NO}_{3}-\right)$, Fosforo $(\mathrm{P})$ y Potasio $(\mathrm{K})$, en las muestras, tres y cuatro de acuerdo al análisis, es alta con valores que van de $\mathrm{NO}_{3-}$ de 60 a 63.3, $\mathrm{P}$ con 60 a 70 y $\mathrm{K}$ de 1 a 2.5, mientras que uno y dos muestran deficiencia en Nitratos en un nivel medio (40-50 ppm). El contenido de $\mathrm{P}$ en las muestras de suelos indico para todas las muestras que se encuentra en niveles altos en un rango de 60 a 79.6 ppm.

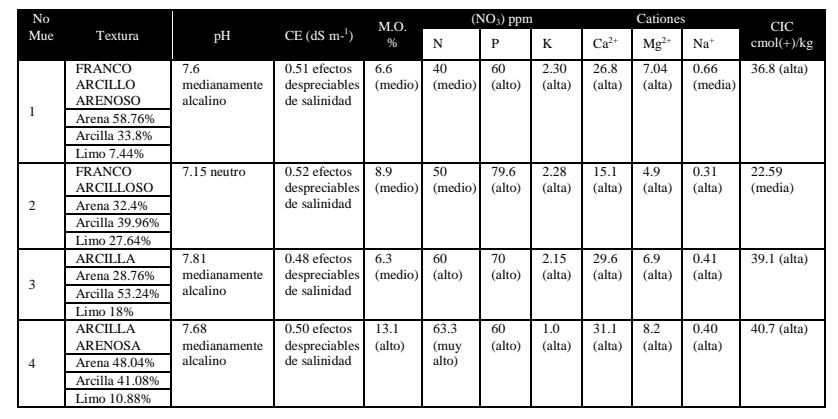

Tabla 1 Análisis físico-químico de muestras del suelo en la comunidad de Cuesta Blanca

Fuente: Elaboración propia con datos de estudios de suelos enviados a laboratorio

\begin{tabular}{|l|r||r|r|}
\hline & \multicolumn{1}{|c|}{ Maíz } & \multicolumn{1}{c|}{ Frijol } & \multicolumn{1}{c|}{ Cebada } \\
\hline Ingreso Total (\$/ha): & $\$ 4,963.00$ & $\$ 8,563.00$ & $\$ 1,813.00$ \\
\hline Consumo Intermedio (\$/ha) & $\$ 1,985.00$ & $\$ 2,346.00$ & $\$ 1,685.00$ \\
\hline Valor agregado (\$/ha) & $\$ 2,978.00$ & $\$ 6,218.00$ & $\$ 128.00$ \\
\hline Remuneración mano de obra, tierra y agua (\$/ha) & $\$ 2,500.00$ & $\$ 2,269.00$ & $\$ 2,431.00$ \\
\hline Remuneración absoluta al capital (\$/ha) & $\$ 478.00$ & $\$ 3,949.00$ & $\$-2,303.00$ \\
\hline Remuneración Relativa al capital $(\%)$ & $11 \%$ & $86 \%$ & $-54 \%$ \\
\hline Costos $(\$ / h a)$ & $\$ 4,484.00$ & $\$ 4,613.00$ & $\$ 4,116.00$ \\
\hline Ganancias (\$/ha) & $\$ 478.00$ & $\$ 3,949.00$ & $\$-2,303.00$ \\
\hline
\end{tabular}

Tabla 2 Estructura del Ingreso, Costos y Ganancias por hectárea de los cultivos en el Cardonal (Incluyendo tierra)

Fuente: Elaboración propia con datos obtenidos en campo
Para el elemento $\mathrm{K}$ todas las muestras se encuentran en niveles altos de 1 a $2.30 \mathrm{cmol}$. En general, al considerar niveles altos y medio de los elementos esenciales, se observó que no existe deficiencia importante en el conjunto de parcelas.

Con respecto a $\mathrm{Ca}^{2+} \mathrm{Na}^{+}$y $\mathrm{Mg}^{2+}$ se encuentran en niveles altos, más solo en la primera muestra con un nivel medio de $\mathrm{Na}^{+}$con $0.66 \mathrm{cmol}$; sin embargo, esto no constituye un riesgo de salinidad en las parcelas estudiadas (Rechard, 1985). La capacidad de intercambio catiónico (CIC), presenta niveles altos en las muestras uno, tres y cuatro que van del 36.8 hasta 40.7 y la parcela tres de $39.1 \mathrm{cmol}$. Las condiciones de intemperismo y el manejo agrícola de las parcelas han coadyuvado a predeterminar los valores de la CIC resultantes, lo que favorece el flujo de iones en las micelas coloidales durante su hidratación, esto favorece que el flujo de nutrimentos y su reserva se lleve a cabo eficientemente (Kononova, 1982).

\section{Producción y rentabilidad de los cultivos en la comunidad de Cuesta Blanca}

El maíz, frijol y cebada son cultivos de importancia económica para la comunidad de Cuesta Blanca y para el mercado regional debido a que son productos de consumo básico para la alimentación de las familias, así como la semilla de dichos cultivos para la siguiente temporada.

La Tabla 2 muestra los ingresos promedio que generan los cultivos empleados en la comunidad: el frijol es el cultivo que genera un mayor ingreso por hectárea $\$ 8,563.00$, superior al ingreso que generan los cultivos de maíz con $\$ 4,963.00$ y cebada $\$ 1,813.00$, debido al precio más alto que se tiene por el producto; sin embargo, el ingreso generando por la cebada no es suficiente para pagar los costos que involucra su producción, generando una pérdida de \$2,303.00 hasituación generada por la no comercialización del producto, ya que es utilizado como forraje para el ganado. Al desglosar el ingreso en sus componentes principales, es decir, consumo intermedio y valor agregado, la cebada presenta la mayor derrama económica al interior de la comunidad, en términos porcentuales, al gastar el $93 \%$ de sus ingresos $(\$ 1,685)$ en la compra de insumos; sin embargo, genera apenas $7 \%$ de valor agregado. 
Por el contrario, el frijol genera la mayor proporción de valor agregado (73\%), haciendo una derrama al interior de la comunidad de apenas $27 \%$ del ingreso lo cual equivale a $\$ 2,346.00 \mathrm{ha}^{-1}$.

En cuanto a la remuneración relativa del capital, que resulta de dividir la remuneración absoluta al capital entre la suma del consumo intermedio más la remuneración de la mano de obra, tierra y agua, por cada peso invertido en la producción, el frijol genera 86 centavos de valor agregado; mientras que, para el caso de la cebada esta relación indica que por cada peso invertido se están perdiendo 54 centavos del valor agregado que se genera en la producción. De acuerdo con estos resultados el frijol es el cultivo que generó mayor ganancia, con respecto a los otros dos cultivos. La situación de la cebada como actividad económica con enormes pérdidas pudiera minimizarse si las personas que la desarrollan, cosecharan el grano y lo comercializaran. Cabe destacar que los resultados muestran que los rendimientos de los cultivos son bajos debido a factores de tipo climáticos y de suelos.

\section{Aplicación de las alternativas de conservación y mejoramiento de la fertilidad de los suelos de la comunidad}

Para las alternativas que se están proponiendo aplicar para la conservación del suelo en la comunidad de Cuesta Blanca, son dos:

Presa de rama acomodada

La aplicación de presas en rama tiene la finalidad de controlar la erosión, reducción de la velocidad de escurrimientos y retener azolves arrastrados por el agua en temporadas de lluvia en zonas de laderas con pendientes pronunciadas. Como efecto de lo anterior atenderá problemas del efecto de la erosión hídrica y detener el crecimiento de cárcavas y permitiendo la acumulación de sedimentos favorables para el establecimiento de cobertura vegetal. La implementación de presas de ramas se determinó como propuesta para el problema de erosión debido a que en la comunidad los límites de la misma están dados por las barrancas próximas a las zonas de cultivo en donde se presentan pequeños escurrimientos que forman cárcavas de mínima dimensión pero que pueden ser atendidas a través de esta técnica de conservación.
Se identificaron previamente los espacios donde eran requeridas las presas debido al avance de las cárcavas, ubicadas mediante el recorrido de campo, así como el de los perfiles de elevación dibujados sobre el polígono de la comunidad en el que se mostraron pendientes que van desde un $2 \%$ hasta un $77 \%$ (Figura 2).

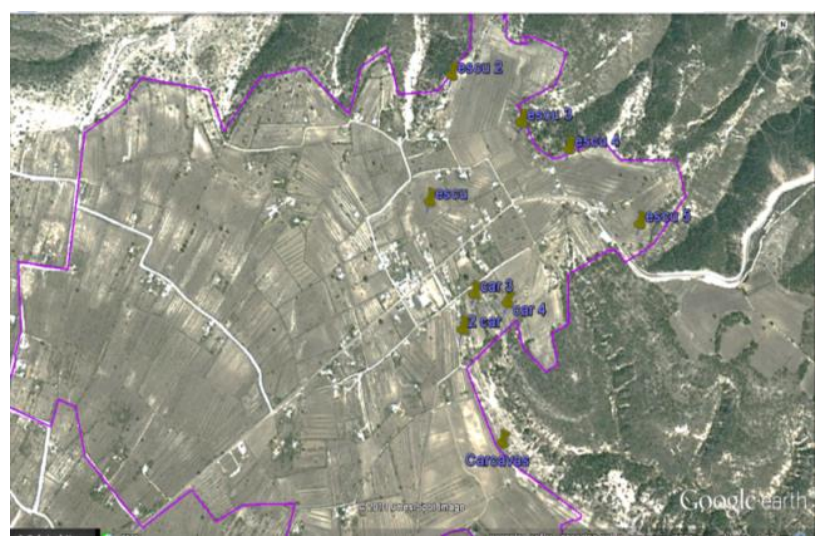

Figura 2 Escurrimientos y cárcavas en la zona de estudio Fuente: Elaboración propia con Google Earth $\odot$

El costo de aplicación de las alternativas de solución es muy importante ya que se tiene que considerar la colocación de los materiales en el sitio de la obra, el transporte, acarreo y la mano de obra para la construcción. Estos costos son variables de acuerdo con la localización del sitio por trabajar y las vías para movilizar los materiales a utilizar. Por lo que se tienen que tomar en cuenta para realizar las acciones que tengan el menor costo y la mayor eficiencia en el control de azolves.

El costo total calculado por presa de rama acomodada fue de $\$ 3,672.00$, de los cuales $\$ 2,422$ lo conforman los materiales para presa de ramas, y $\$ 1,250.00$ son costos de la implementación de estas presas, si se consideran un total de aproximado de 9 presas, entonces el costo total es de $\$ 33,048.00$. Estos costos son para presas en rama de dos metros de largo por un metro de alto y 0.2 metros de empotramiento. Determinado el gasto generado para una presa en rama se considera viable debido a que es una obra que no se desarrollara en años posteriores. Con esta actividad junto con la aplicación de biofertilizantes se pretende generar condiciones favorables para la actividad agrícola de producción de cultivos tradicionales de la región. 
b)

\section{Abonos fermentados tipo Bocashi}

El costo de producción de abono orgánico por hectárea es de $\$ 2,680.00$. Los materiales son propios de la región por lo cual no se tendría que trasportar desde grandes distancias, sus costos no son elevados y la disponibilidad es la necesaria para la aplicación por hectárea. Para ingredientes como; estiércol, tierra, pulque y piloncillo son productos que implica cierto transporte; para el carbón cisco y la ceniza solo implica la mano de obra para recolectar los materiales, solo el maíz en mazorca implica mano de obra y costo de material.

Para el primer año se propone aplicar 3 dosis por hectárea, esto debido a las condiciones de marginalidad en los suelos, además de considerar que se seguirán cultivando los terrenos mientras los nutrientes y materiales de los abonos se incorporan a los suelos a través de la mineralización de los elementos. Para el año tres las condiciones del suelo ya habrán mejorado debido a la incorporación de dicho abono y de tener el tiempo necesario para la descomposición de los elementos del mismo; para el quinto año será de una aplicación al año, debido a que se busca compensar lo que en el año agrícola anterior que se desgasto por el cultivo.

\section{Evaluación económica de la implementación de las técnicas de conservación y mejoramiento de fertilidad del suelo}

Se evaluaron 3 técnicas de conservación para el mejoramiento del suelo de la comunidad de Cuesta Blanca, las cuales fueron:

a)

Rendimientos con medidas de
conservación

Se propone utilizar medidas que generen la disponibilidad de nutrimentos en el suelo, así como en zonas con laderas se implementen presas con ramas lo que llevará a una afectación a los costos empleados debido a estas dos medidas. De igual manera se verán afectados los rendimientos, así como la calidad de los suelos; la aplicación del biofertilizante implica la utilización de mayor mano de obra y el uso de productos de la región que son parte del consumo intermedio para el primer año y para el segundo, y así consecutivamente en los siguientes años.
Los beneficios ecológicos esperados se presentan junto con los rendimientos, como se muestra Grafica 1.

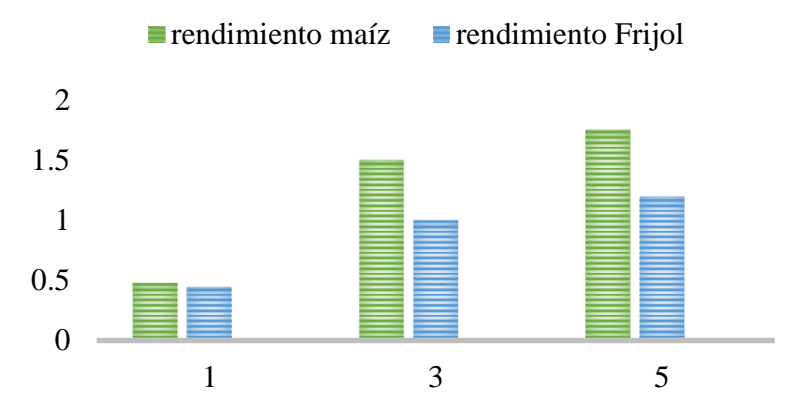

Gráfica 1 Rendimientos esperados de cultivos con implementación de técnicas de conservación (ton)

Fuente: Elaboración propia con datos obtenidos en campo

Para el primer año, el incremento pudiera ser muy poco debido a la asimilación de los micronutrientes disponibles, pero manteniendo la actividad complementaria de aplicación del biofertilizante, llevara a mantener condiciones óptimas para los cultivos empleados en los años posteriores, donde se esperan incrementos importantes.

\section{b) Costos de producción}

Los costos de producción de maíz con la aplicación de técnicas de conservación, representan un incremento de más del $90 \%$ de los costos en cuanto a los factores internos para el primer año en el cual se aplican en tres ocasiones el abono orgánico fermentado y la aplicación de las presas en rama, para el tercer año será de un $86 \%$ con tan solo dos aplicaciones de los abonos sin contabilizar las presas en ramas y para el quinto tan solo una aplicación de abonos, esto representar un $80 \%$ de los costos.

Para el caso del cultivo del frijol con técnicas de conservación, los costos de producción se ven afectados de igual manera en los factores internos por los mismos factores que en el cultivo de maíz, representando un 90\% de los costos en factores internos para el primer año de aplicación, para el tercer año un $83 \%$ y para el quinto año solo el $77 \%$ de estos costos. 
Para la cebada, los costos de producción con técnicas de conservación, se incrementa en el año uno hasta un $93 \%$ en el apartado de factores internos por las mismas condiciones de aplicación de las técnicas en el cultivo de maíz y frijol, para el tercer año el incremento representa el $88 \%$ y para el quinto año con el $81 \%$.

La aplicación de abono orgánico como actividad de conservación, es el principal detonante para elevar los costos de producción siendo el primer año donde se hace una gran inversión debido al número de aplicaciones, así como la implementación de las presas en rama. Los factores internos representan en el análisis de la actividad agrícola bajo los tres cultivos un porcentaje de menos del $70 \%$ y ya con la aplicación de las técnicas en el primer año representan no menos del 90\%, representando un impacto en la inversión.

\section{c) Rentabilidad}

El beneficio económico para el primer periodo en el cual los rendimientos siguen siendo los mismos que cuando no se aplicaban las técnicas de conservación, representan pérdidas para todos los cultivos, $69 \%$ para maíz, $48 \%$ para frijol y $89 \%$ para la cebada, debido al proceso de desintegración de la materia y su incorporación al suelo. Para el tercer año las ganancias ya representan números positivos para maíz y frijol debido a que los rendimientos son mayores, así como un nivel de costos menor que en el primer año (Gráfica 2).

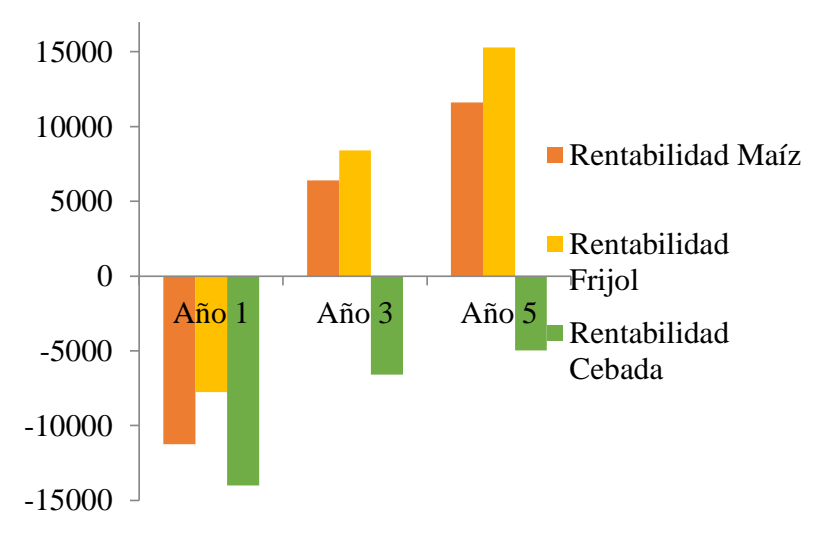

Grafica 2 Rentabilidad de actividad agrícola bajo técnicas de conservación (\$)

Fuente: Elaboración propia con datos obtenidos en campo
Para el cultivo de cebada la rentabilidad mantiene una negatividad debido a que solo se aprovecha como forraje, siendo éste el único que represente pérdidas para el quinto año ya que el frijol y maíz retoman nuevamente números positivos en cuanto a rentabilidad se refiere, esto debido a los incrementos que pudieran desprenderse a partir de la incorporación de dichas técnicas elevando rendimientos y como resultado, mayor ingreso al productor (Tabla 3).

\begin{tabular}{|l|r|r|}
\hline \multicolumn{1}{|c|}{ Ganancia neta } & \multicolumn{1}{c|}{ Año 3 } & \multicolumn{1}{c|}{ Año 5 } \\
\hline Maíz & $\$ 6,405.75$ & $\$ 11,619.08$ \\
\hline Frijol & $\$ 8,418.07$ & $\$ 15,293.90$ \\
\hline Cebada & $-\$ 6,588.33$ & $-\$ 4,983.33$ \\
\hline \multicolumn{3}{|c|}{ Rendimientos (ton/ha) } \\
\hline Maíz & 1.5 & 1.75 \\
\hline Frijol & 1 & 1.2 \\
\hline Cebada & 70 (pacas) & 100 (pacas) \\
\hline
\end{tabular}

Tabla 3 Ganancias y rendimientos resultantes de la proyección a la incorporación de las técnicas de conservación

Fuente: Elaboración propia con datos obtenidos en campo

De esta forma con la aplicación de las técnicas de conservación y recuperación de la fertilidad de los suelos en la comunidad elevando la producción de maíz en un aproximado de una tonelada y media por hectárea y hasta una en frijol para el quinto año de aplicación del abono orgánico, de igual forma podría ser perceptible el beneficio económico desde el tercer año de aplicación de dichas técnicas, generando de igual forma fertilidad en los suelos. Estos datos son bajo la estimación de la MAP a precios de 2012 lo cual podría generar variaciones para otros años.

\section{Conclusiones}

Los análisis de suelo muestran que los suelos están dentro del grupo de textura media, unos de tipo franco arcillo arenoso y franco arcillo, y otros fina arcilla arenosa y arcilla. Con un $\mathrm{pH}$ medianamente alcalino que oscila en promedio a 7.56 que indica un estatus medianamente alcalino. Condición que pudiera generar problemas de adquisición de elementos contenido en los suelos, generando problemas con los rendimientos. En general los resultados muestran condiciones favorables para los cultivos empleados en la región debido a que no se encuentra problemas de salinidad severos y de empobrecimiento de nutrientes. 
De los tres cultivos que se trabajan en la comunidad; el maíz es el de mayor importancia debido a las utilidades de dieta para las familias de la región, así como de la subutilización como forraje para el ganado de traspatio. El frijol es el cultivo que genera mayores ganancias para los agricultores de la comunidad debido a los niveles de rendimiento y el precio en el mercado.

Para atender el problema de empobrecimiento de nutrientes en los suelos así como el de evitar la erosión y problemas de arrastre de suelos se plantean dos alternativas de conservación de suelos para la comunidad de Cuesta Blanca: la implementación de presas de rama acomodada, cuya implementación empata con las condiciones de la localidad por los materiales a necesitar y porque a pesar de que las cárcavas son significativas año con año generan un arrastre importante de sedimentos que son vitales para la actividad agrícola. Otra alternativa es la utilización del biofertilizante de tipo Bocashi que es favorable debido a su existencia y su bajo costo, factores que lo hacen una alternativa para el enriquecimiento de los suelos agrícolas de la comunidad.

La aplicación de abonos orgánicos en los primeros años genera una inversión fuerte debido al inicio se aplican tres dosis de abono por las condiciones actuales, en el segundo y tercero solo se aplicarán dos y para el quinto solo una. Esta condición permitirá a los suelos mantener su fertilidad por mayor tiempo a consecuencia de un desgaste mínimo y a su compensación año tras año.

La mejora en rendimientos es notable al tercer año de aplicación lo que posibilitaría al maíz sobre pasar la media tonelada de igual forma el cultivo de frijol y la cebada con un incremento de hasta 70 pacas. Si bien se incrementan los costos de producción se elevan la rentabilidad de la actividad agrícola viéndose favorecida con mayores utilidades, generando así la reincorporación de la actividad agrícola a los pobladores de la comunidad.

\section{Referencias}

Becerra Moreno, Antonio (1999). Escorrentía, erosión y conservación del suelo. Primera edición en español, DR C Universidad Autónoma Chapingo. México 376 p.
Cueto J. (2010). Compilador Reunión nacional de innovación agrícola, Campeche, México

Claude Q. (1960). La erosión de suelo y su control. Omega S. A. Barcelona 437 p.

Cruz S. (1986). Abonos Orgánicos, Universidad Autónoma Chapingo, México, pág. 104-122.

De la Rosa D. (2008). Evaluación Agroecológica de Suelos, CSIC-IRNAS, Sevilla, pág. 404

FAO, ISRIC and ISSS (1998). World reference base for soil resources. Rome: Food and Agriculture Organization of the United Nations

Foth, Henry (1996). Fundamentos de la ciencia del suelo. 6ta reimpresión. Compañía Editorial Continental S.A. de C.V., 433 p. México.

Fuentes. L. (1999). Manual práctico de manejo de suelos y de fertilizantes, edición MundiPrensa México.

Hudson N. (1982). Conservación de suelo, editorial Reverte, México.

Instituto Nacional de Estadística y Geografía (INEGI, 2012). Aspectos generales del territorio mexicano. Recursos naturales. Edafología. México. Disponible en: http://mapserver.inegi.org.mx.

Jiménez R. (1998). Agricultura sostenible, edición Mundi-prensa, México.

Kononova M. (1982). Materia orgánica del suelo, su naturaleza, propiedades y métodos de investigación, Oikos-tau Barcelona España. Pág. 157-165.

López F. (2002). Erosión y desertificación, heridas de la tierra. Nivola libros, España.

Morgan P.R.C. (1997). Erosión y conservación del suelo $1^{\mathrm{a}}$ edición. Editores Mundi-Prensa. México 343 p.

Oldeman L.R. (1988). Guidelines for general assessment of the status of human-induced soil degradation. Working paper 88/4. International Soil Reference and Information Centre (ISRIC), Wageninen, 151p. 
Porta L. López M. \& Poch R. (2008). Introducción a la edafología, uso y protección del suelo. Mundi-Prensa, Madrid, Barcelona 443 p.

Sánchez Ballesta Juan Pedro. (2002). “Análisis de la rentabilidad de la empresa" Análisis contable. Versión electrónica.

Secretarias de Educación Pública (SEP) (1983). Manuales de Educación Agrícola, Preparación de Tierras Agrícolas, México.

Secretaria de Educación Pública (SEP) (2010). Manuales para la educación agropecuaria, Suelos y Fertilización. Trillas México.

Secretaría de Medio Ambiente, Recursos Naturales y Pesca (SEMARNAP). y CP. (2003). Evaluación de la degradación del suelo causada por el hombre en la República Mexicana, escala 1:250 000. México.

Secretaría de Medio Ambiente, Recursos Naturales y Pesca (SEMARNAP) (2008). Compendio de Estadísticas Ambientales. México.

Secretaría de Medio Ambiente, Recursos Naturales y Pesca (SEMARNAP) s/f. Centro Piloto "El Dexthi" Hidalgo. Sistematización y evaluación. Subsecretaría de Recursos Naturales. Dirección General de restauración y conservación de suelos.

Simpson K. (1991). Abonos y estiércoles, editorial Longman Group Limited, España.

Sparovek, G., Teramoto, E.R., Toreta, D.M., Rochele, T.C. y Shayer, E.P.M. (1990). Erosao simulada e produtividade do milho. In: Congresso Brasileiro e Encontro Nacional de Pesquisa sobre Conservaçao do Solo. VIII Londrina, 1990. Anais. Londrina, Sociedade Brasileira de Ciencia do Solo, pág. 95.

Velazco Molina, Hugo A. (1983). Uso y manejo del suelo. Limusa S. A., México 191 p. 